\title{
Three-dimensional verification of volumetric measurements and relationships between the condyle and the rest of the mandible; a novel approach
}

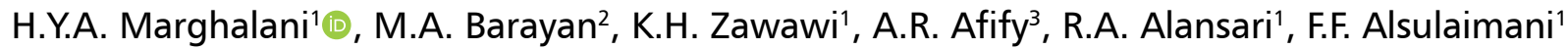 \\ 'Orthodontic Department, Faculty of Dentistry, King Abdulaziz University, Jeddah, Saudi Arabia \\ 2Oral Basic Science, Oral and Maxillofacial Radiology Department, Faculty of Dentistry, \\ King Abdulaziz University, Jeddah, Saudi Arabia \\ ${ }^{3}$ Orthodontic Department, Faculty of Dentistry, Mansoura University, Mansoura, Egypt
}

[Received: 29 June 2020; Accepted: 21 August 2020; Early publication date: 2 September 2020]

\begin{abstract}
Background: Few studies have investigated the volumetric relationship between the condyles and the mandible bilaterally.

Materials and methods: Condylar and mandibular segmentations from a sample of cone-beam computed tomography for 37 individuals were selected. T-test, correlation and linear regression analyses were performed to assess the relationships between the volumes of the condyles and mandible.

Results: The volume of the condyles and the mandible was significantly different between genders $(p<0.05)$. There was a significant but moderate correlation between the volumes of the condyle and the rest of the mandible on the same side $(p<0.01)$. A regression analysis model demonstrated that condylar volume is related to the volume of the mandible.

Conclusions: The relationship between the condylar volume and the rest of the mandible was found to be moderate. The relation between the condylar volume and mandibular volume is described by the regression equations for each side of the jaw. Sexual dimorphism exists in condylar and mandibular volumes. (Folia Morphol 2021; 80, 3: 650-656)
\end{abstract}

Key words: cone-beam computed tomography, volume, condyle, mandible, segmentation

\section{INTRODUCTION}

The mandible has many vital roles for humans, such as mastication, and facial aesthetics [1]. Moreover, the relationship between the mandible and the condyle has been the focus of some studies $[4,16]$. Most of the researches that studied the mandible utilised two-dimensional imaging such as cephalometric radiographs which have limitations in accuracy and reliability $[10,11,20]$.
Condylar growth was suggested to be regulated by the function of the temporomandibular joint [23]. An example of the effect of function on growth is the presence of structural asymmetry due to functional shift causing more growth on the protruded side [23]. Also, mandibular asymmetry was found in patients suffering from unilateral anterior disc displacement that caused the condylar height to be shorter on the affected side [25]. 

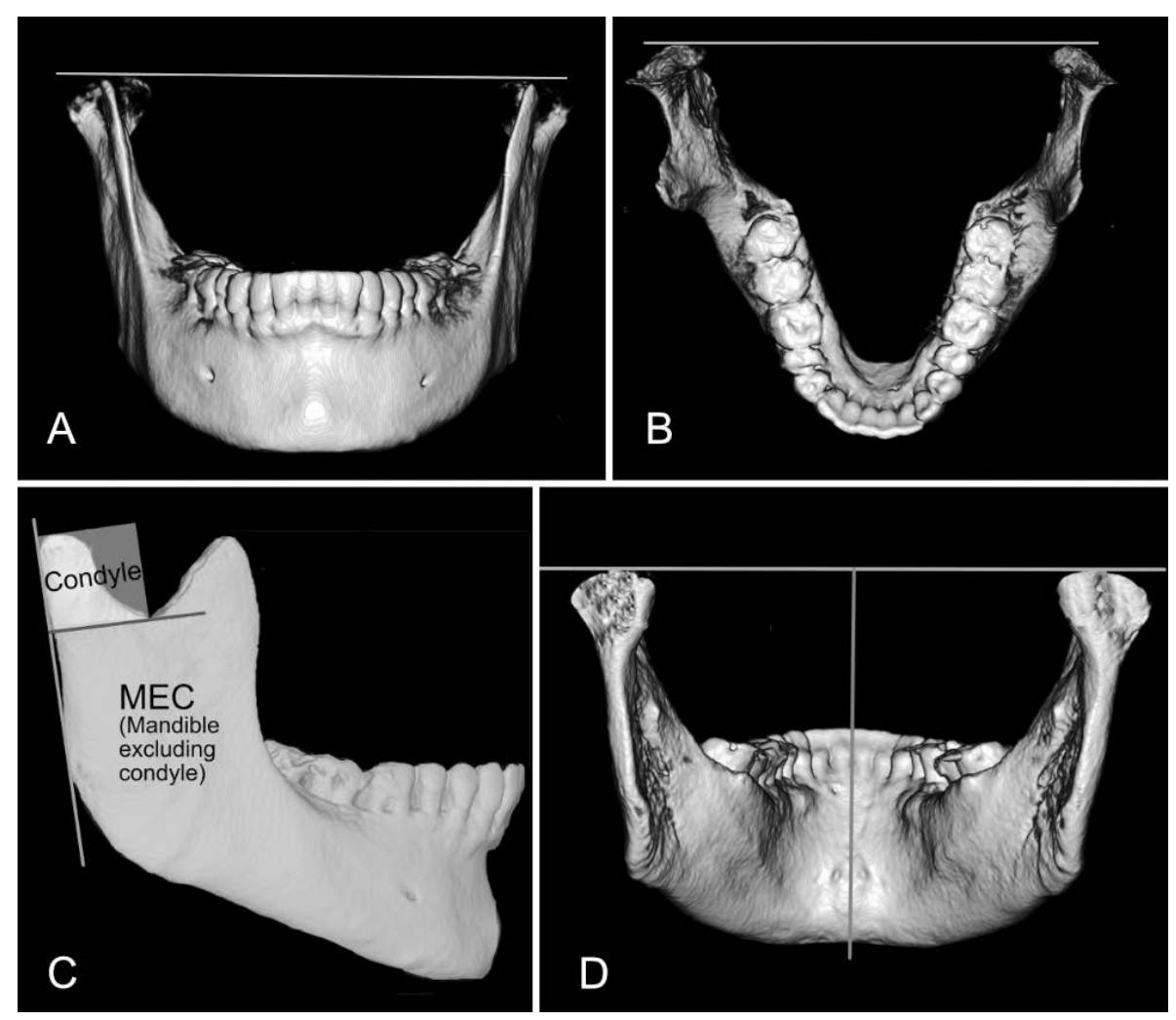

Figure 1. A. Orientation of the mandible in the coronal plane; B. Orientation of the mandible in the transverse plane; C. Segments of mandible used for volumetric measurement. The condyle is segmented out of mandible using landmarks shown; $\mathbf{D}$. Identification of mandibular midline using line passing above condyles and perpendicular line to lingual foramen between genial tubercles.

Three-dimensional imaging using cone-beam computed tomography (CBCT) has become an integral part of dentistry [9]. High-resolution images of the craniofacial structures enable accurate volumetric and linear measurements $[5,21]$. In dentistry, CBCT is commonly used before the placement of dental implants, assessment of impacted teeth and temporomandibular joint [2]. CBCT is also used to measure the volume of craniofacial structures $[5,7,21]$.

To the best of our knowledge, few studies, have investigated the volumetric relationship between the condyles and the mandible bilaterally. The main objective of this study was to assess the relationship between the volume condyle and the mandible, using the CBCT segmentation method. A secondary objective was to evaluate if the mandibular volume can be predicted by the volume of the ipsilateral condyle.

\section{MATERIALS AND METHODS}

This study was approved by the Research Ethics Committee (No. 084-09-18). The sample consisted of consecutive patients who had $\mathrm{CBCT}$ imaging at the Radiology Department at the Faculty of Dentistry with the following inclusion criteria: 1) patients 17 years or older, 2) adequate resolution of the CBCT images of all the mandible and condyles, 3) no craniofacial deformity, pathology, fractures or jaw surgery, and 4) no dental anomalies such as supernumerary, fusion or impacted teeth.

Using the G*Power software (Heinrich Heine Universitat Dusseldorf, Germany), a priori power analysis was performed to calculate the appropriate sample size. For the correlation analysis (2-tailed) with alpha set as 0.05 and power of 0.80 , the required total sample size was 13 individuals. While, with alpha set as 0.01 and power of 0.95 , the required total sample size was 26 individuals.

Cone-beam computed tomography images were taken using iCAT (Imaging Sciences International, Hatfield, PA, USA) with settings of $120 \mathrm{Kvp}$ and $5 \mathrm{~mA}$, and segmented separating the mandible from the skull using OnDemand software (build 1.0.10.7462 by cybermed, Seoul, Korea). The mandible was oriented using intercondylar line tangent to the most superior and posterior aspect of condyles and was parallel to a horizontal line in the coronal and transverse planes (Fig. 1A, B). 
Table 1. Comparison of total condyle and mandible excluding condyle (MEC) volumes between males and females

\begin{tabular}{lccc}
\hline & Males $(\mathbf{n}=\mathbf{1 3})$ & Females $(\mathbf{n}=\mathbf{2 4})$ & $\mathbf{P}$ \\
\hline Total condyle volume & $5,757.7(1441.6)$ & $4,671.9(1205.1)$ & 0.02 \\
Total MEC volume & $75,177.4(10976.8)$ & $61,226.0(10905.4)$ & 0.001 \\
\hline
\end{tabular}

Data are presented as mean (standard deviation) in $\mathrm{mm}^{3}$

Table 2. Comparisons between right and left condyle and mandible excluding condyle (MEC) volumes for the total sample

\begin{tabular}{|c|c|c|c|}
\hline & \multicolumn{2}{|c|}{ Volume [mm³] } & \multirow[t]{2}{*}{$\mathbf{P}$} \\
\hline & Right (n = 37) & Left ( $n=37)$ & \\
\hline Condyle & 2496.5 (708.7) & $2556.7(719.5)$ & 0.718 \\
\hline MEC & $33201.3(6687.2)$ & $32926.51(6120.6)$ & 0.854 \\
\hline
\end{tabular}

Data are presented as mean (standard deviation)

Digital segmentation of the condyle was done using landmarks that were derived from the guidelines of the AOCMF classification system [17]. A tangent line to the most posterior part of the mandible touching both the condylar and gonial regions was drawn. From this line, another perpendicular line passing the most inferior point of the sigmoid notch was formed. The volume above the sigmoid notch line was the condylar volume; while the remaining volume was the mandible and was referred to as "mandible excluding condyle" (MEC) (Fig. 1C). To segment the mandible into two halves, a line perpendicular to the intercondylar line and passing through lingual foramen between the two genial tubercles in the anterior lingual aspect of the mandible (Fig. 1D). This perpendicular line was found to be a stable midline structure for the mandible [12].

All measurements were performed using OnDemand software by one investigator with more than 5 years of experience from the Maxillofacial Radiology Department. The measurements were performed twice with at least 2 weeks interval to test the intra-examiner reliability. The interclass correlation coefficient showed that the intra-examiner reliability ranged from 0.967 to 0.994 .

\section{Statistical analysis}

Data on the condylar and MEC volumes were collected and analysed using Statistical Package for Social Sciences (SPSS Version 25, Armonk, NY: IBM Corp., USA). The Shapiro-Wilk test showed a normal distribution of the data. Comparisons between variables were performed using the t-test. Pearson correlation coefficient and linear regression analysis were performed. A significant level was set at $p<0.05$.

\section{RESULTS}

The sample included 74 segmentations for 37 patients, 13 (35.1\%) males and 24 (64.9\%) females. The mean age was $29 \pm 13$ years for males and $40 \pm$ \pm 14 years for females. There was a significant difference in age between sexes $(p=0.031)$.

Table 1 shows comparisons of the total condyle and MEC volumes between males and females. The total condylar volume and MEC were significantly larger in males than in females $(p=0.02$ and 0.001 , respectively).

Table 2 shows comparisons between right and left condylar and MEC volumes for the total sample (combined males and females). The volume of the left condyles was larger (mean $=2556.7 \mathrm{~mm}^{3}$ ) than the right condyle $\left(\right.$ mean $\left.=2496.5 \mathrm{~mm}^{3}\right)$; however, the difference was not statistically significant ( $p=0.718$ ). Interestingly, the right MEC volume (mean $=33201.3 \mathrm{~mm}^{3}$ ) was larger than the left MEC (mean $=32926.5 \mathrm{~mm}^{3}$ ), but this difference was also not statistically significant ( $p=0.854$ ).

Table 3 shows in detail the comparisons between gender and sides of the condyle and MEC volumes. There were no significant differences between the right and left volumes of the condyles in males or females $(p>0.05)$. However, the right and left condyles were statistically significantly different between males and females, $p=0.024$ and 0.026 , respectively. MEC volume was not different between the right and left in both males and females, $p>0.05$. However, males had a significantly larger right and left MEC compared to females, $\mathrm{p}=0.001$.

There was a strong correlation between the volumes of the right and left condyles and the right and left MEC ( $r=0.859$ and 0.972 , respectively). The cor- 
Table 3. Comparisons between gender and sides of condyle and mandible excluding condyle (MEC) volumes.

\begin{tabular}{|c|c|c|c|c|}
\hline & Males ( $n=13$ ) & Females $(n=24)$ & Difference & $\mathbf{P}$ \\
\hline \multicolumn{5}{|l|}{ Condyle } \\
\hline Right & $2,848.6(649.1)$ & $2,305.8(676.9)$ & 542.8 & 0.024 \\
\hline Left & $2,908.55(827.4)$ & $2,366.18(587.6)$ & 542.4 & 0.026 \\
\hline Difference & 59.9 & 60.4 & & \\
\hline$P$ & 0.84 & 0.74 & & \\
\hline \multicolumn{5}{|l|}{ MEC } \\
\hline Right & $37,972.7(5,917.3)$ & $30,616.9(5,646.9)$ & 7355.8 & 0.001 \\
\hline Left & $37,204.7(5,164.0)$ & $30,609.2(5,364.2)$ & 6595.6 & 0.001 \\
\hline Difference & 767.9 & 7.7 & & \\
\hline$P$ & 0.73 & 0.996 & & \\
\hline \multicolumn{5}{|c|}{ Total (Condyle + MEC) } \\
\hline Right & $40,821.3(6361.8)$ & $32,922.7(5884.1)$ & 7898.6 & 0.001 \\
\hline Left & $40,113.3(5,671.4)$ & $32,975.3(5,587.7)$ & 7137.9 & 0.001 \\
\hline Difference & 708.0 & 52.7 & & \\
\hline$P$ & 0.77 & 0.975 & & \\
\hline
\end{tabular}

Data are presented as mean (standard deviation) in $\mathrm{mm}^{3}$

Table 4. Correlations analysis of condylar and mandible excluding condyle (MEC) volumes

\begin{tabular}{lcccc}
\hline & Condyle Right & Condyle Left & MEC Right & MEC Left \\
\hline Condyle Right & & & \\
Condyle Left & $0.859^{* *}$ & & \\
MEC Right & $0.528^{*}$ & $0.535^{*}$ & \\
MEC Left & $0.535^{*}$ & $0.525^{*}$ & $0.972^{* *}$ \\
\hline
\end{tabular}

${ }^{*} p=0.001,{ }^{* *} p<0.001$

relations between the right condyle and right $\mathrm{MEC}$ as well as the left condyle and left MEC were moderate ( $r=0.528$ and 0.525 , respectively) (Table 4$)$.

Linear regression analysis showed that there was a significant linear relationship between condyle volume and the rest of the MEC on each side $(p<0.001)$ (Figs. 2-4). The equation to predict the volume of the right MEC from the right condyle (Condyle Rt) is: Volume of right MEC = 20764.1 + 4.98 (Condyle Rt). For the prediction of left MEC from the left condyle (Condyle Lt) the equation is: Volume of left MEC = $=21508.1+4.47$ (Condyle Lt).

\section{DISCUSSION}

In this study, a volumetric comparison was made between the right and left condyles and the rest of mandible in males and females. There was a significant difference in the total volumes of condyles and MEC between males and females with higher means in males. In the current study, the mean age of fe- males was larger than males. This difference might be due to the larger number of females visiting orthodontics clinics than males in the sample. This study may suggest that sexual dimorphism exists in the volume of the condyles and rest of the mandible in each side in individuals who completed their growth, with males having larger volumes than females. Other studies also confirmed sexual dimorphism in the mandible [3, 24]. The mean maximum bite force in males was shown to be significantly higher than in females [8], which could explain the difference in volume between males and females.

Perfect facial symmetry is usually found in high quality individuals who maintain symmetric facial development even during genetic and environmental stressors [18]. In our study, when comparing the volume of the condyles and MEC between the right and left sides, no differences were found when comparing the total sample or dividing it based on gender (Table 3). This could be attributed to the fact that our sample 


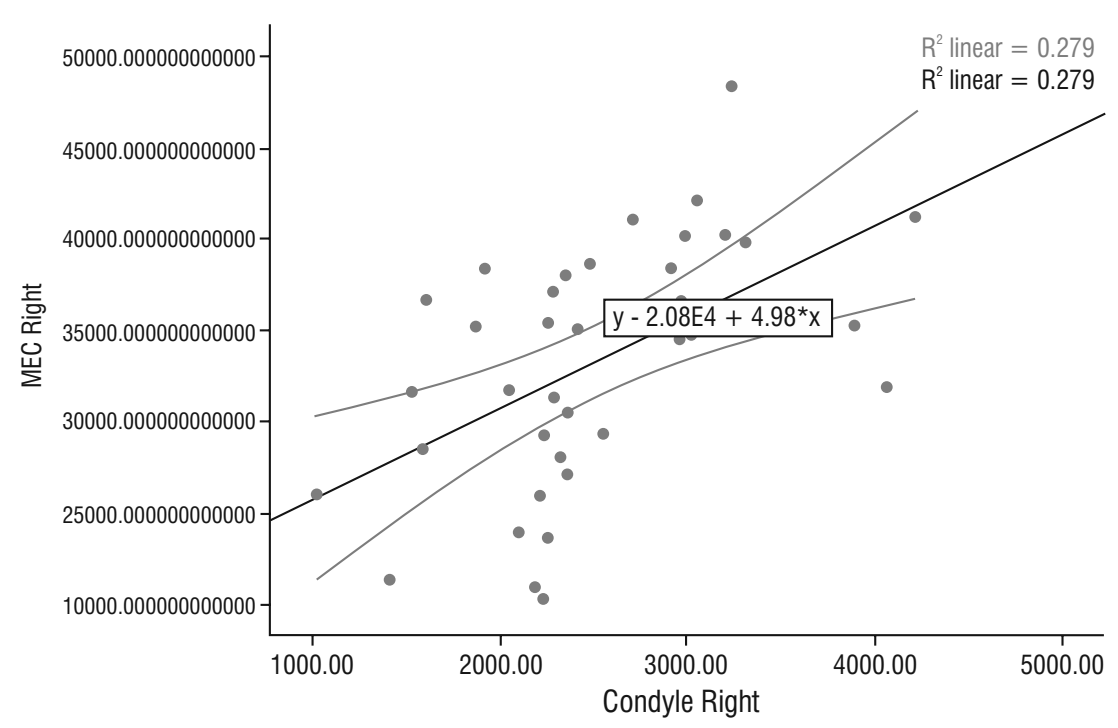

Figure 2. Correlation between right condylar volume and right mandible excluding condyle volume; MEC — mandible exluding condyle.

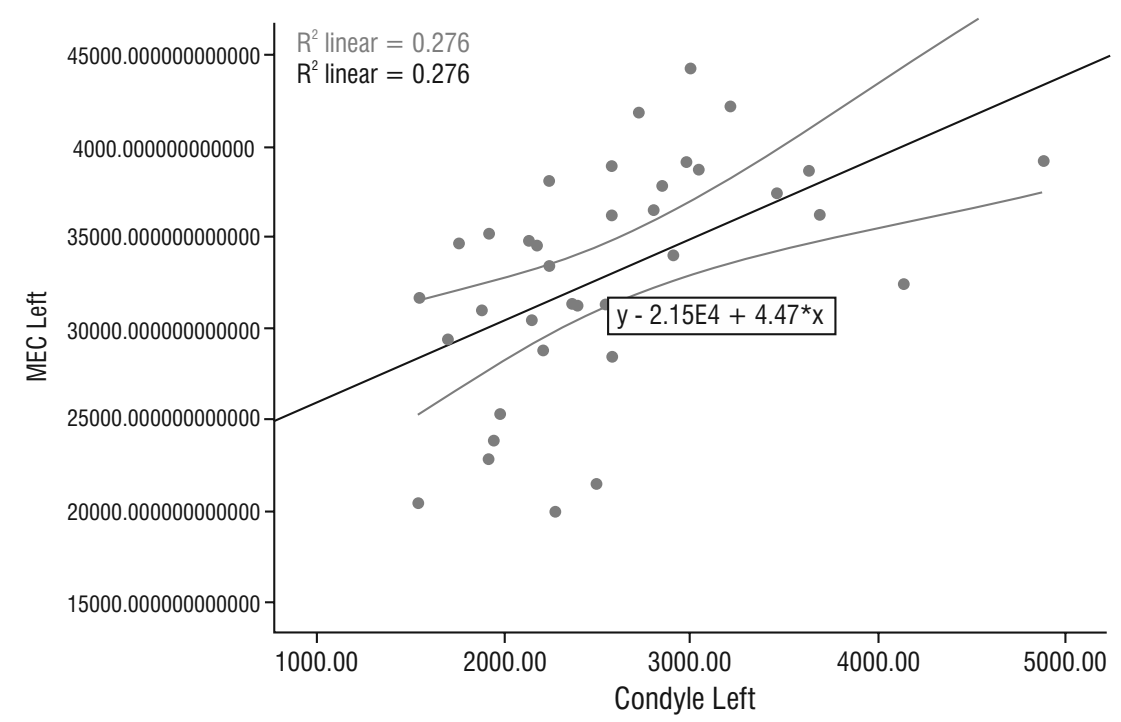

Figure 3. Correlation between left condylar volume and left mandible excluding condyle volume; MEC — mandible exluding condyle.

did not present with significant facial or mandibular asymmetry.

Sella-Tunis et al. [19] found that the function of muscles of mastication, especially the temporalis muscle, in addition to condyles, has a contributing factor to the volume of MEC. This finding might be the cause of larger mean volume in the right MEC but smaller volume in the right condyle than the other side. This finding could be attributed to the fact that most of the patients might be chewing on the right side [22]. Another study was demonstrated by Miyazaki et al. [14] who investigated the effect of muscle activity on the condyle, found that the difference in the lateral activity of the masseter muscle affects the chondro- genesis of the condyle for growing patients. Also, the function of both the masseter and temporalis muscles were found to contribute to the shape of the mandible [19].

In this study, the positive correlation between condyle and mandible is supported by other studies. For example, it was found that the growth of the condyle by itself is related to the vertical and horizontal growth of the ramus [13]. Mandibular growth has been postulated to be affected by the condylar cartilage. According to the functional matrix theory, the mandible grows in response to the soft tissue matrix surrounding it [15]. Moreover, if the condyle was fractured on one side, asymmetry will be caused 


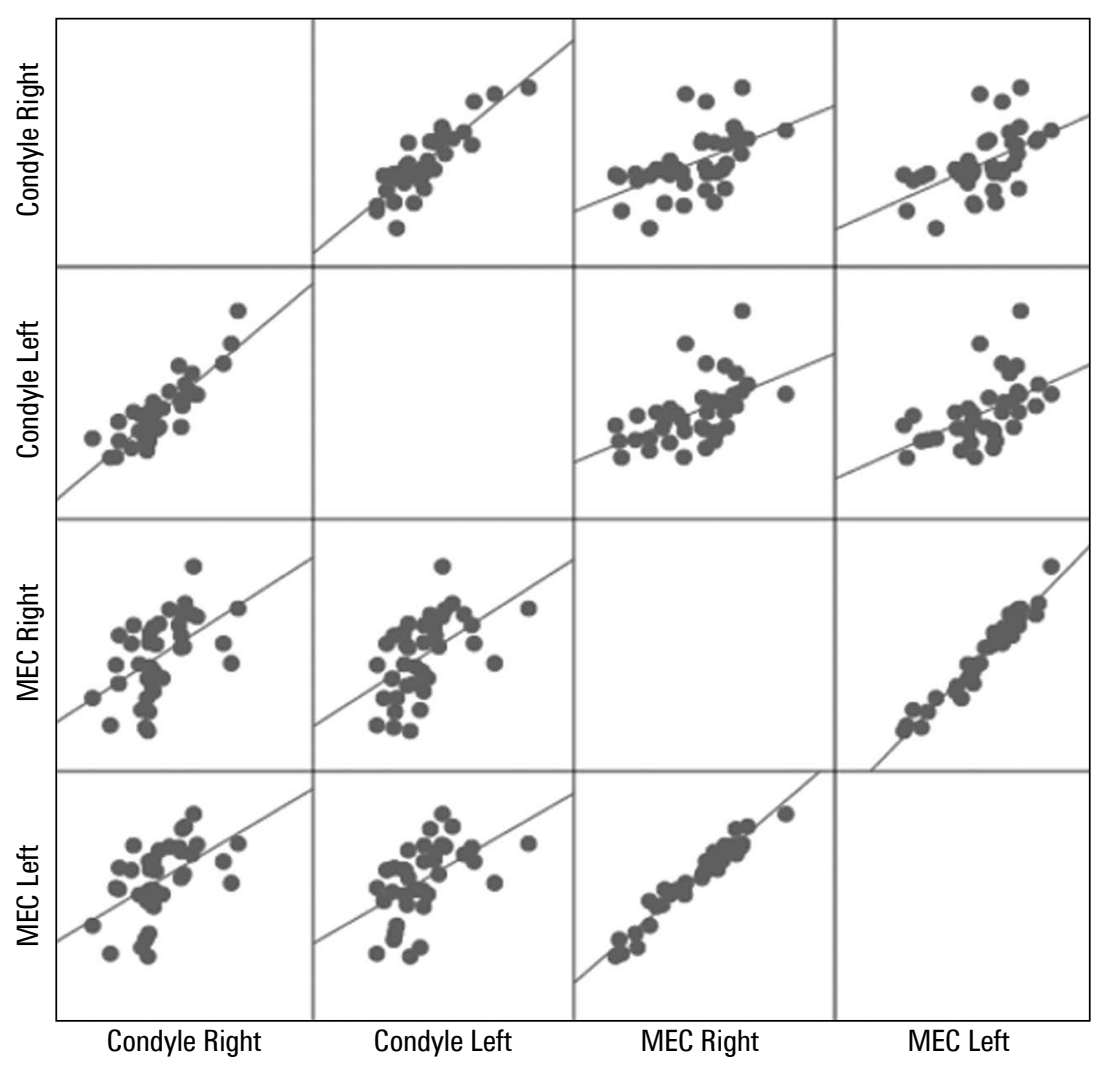

Figure 4. Matrix showing various relationships between condylar and mandible excluding condyle volumes; MEC — mandible exluding condyle.

by the decrease of both the condylar process and the associated side of the mandible [6]. These studies show that there is a relationship between condyle and mandible. Our study analysed this relationship in further detail.

In the current study, the volume of each condyle was found to be related to the volume of the rest of the mandible (MEC) where we found a moderate to strong positive correlation between the condyles and the MEC on each side. Our results are in agreement with Meikle [13].

The linear regression model between the volume of MEC and the condyles demonstrate that condylar volume has an influence on the MEC volume. So, as the condylar volume increases, the MEC volume increases.

The current study has some limitations such as gender and age distribution were not equal. This could be attributed to the stringent inclusion criteria. Future prospective longitudinal multicentre studies with a larger sample size are needed.

\section{CONCLUSIONS}

Within the limitations of this study, we found that sexual dimorphism exists in the volume of the condyles and rest of the mandible in each side in individuals who completed their growth, with males having larger volumes than females. It also confirms using $C B C T$ segmentation that the condyle, at a specific time point, is related to the volume of the rest of the mandible on each side. Also, this study shows that the condylar volume can predict the volume of MEC.

Conflict of interest: None declared

\section{REFERENCES}

1. Choi AH, Conway RC, Taraschi V, et al. Biomechanics and functional distortion of the human mandible. J Investig Clin Dent. 2015; 6(4): 241-251, doi: 10.1111/jicd.12112, indexed in Pubmed: 25044432.

2. De Vos W, Casselman J, Swennen GRJ. Cone-beam computerized tomography (CBCT) imaging of the oral and maxillofacial region: a systematic review of the literature. Int Oral Maxillofac Surg. 2009; 38(6): 609-625, doi: 10.1016/j. ijom.2009.02.028, indexed in Pubmed: 19464146.

3. Fan Yi, Penington A, Kilpatrick N, et al. Quantification of mandibular sexual dimorphism during adolescence. J Anat. 2019; 234(5): 709-717, doi: 10.1111/joa.12949, indexed in Pubmed: 30834524.

4. Farronato M, Cavagnetto D, Abate A, et al. Assessment of condylar volume and ramus height in JIA patients with unilateral and bilateral TMJ involvement: retrospective case-control study. Clin Oral Investig. 2020; 24(8): 2635-2643, doi: 10.1007/s00784-019-03122-5, indexed in Pubmed: 31760476. 
5. García-Sanz V, Bellot-Arcís C, Hernández V, et al. Accuracy and reliability of cone-beam computed tomography for linear and volumetric mandibular condyle measurements. A human cadaver study. Sci Rep. 2017; 7(1): 11993, doi: 10.1038/s41598-017-12100-4, indexed in Pubmed: 28931867.

6. Hu Y, Yang Hf, Li S, et al. Condyle and mandibular bone change after unilateral condylar neck fracture in growing rats. Int J Oral Maxillofac Surg. 2012; 41(8): 912-921, doi: 10.1016/j.ijom.2011.12.036, indexed in Pubmed: 22429642.

7. Huntjens E, Kiss G, Wouters C, et al. Condylar asymmetry in children with juvenile idiopathic arthritis assessed by cone-beam computed tomography. Eur J Orthod. 2008; 30(6): 545-551, doi: 10.1093/ejo/cjn056, indexed in Pubmed: 19054811.

8. Jain V, Mathur VP, Pillai RS, et al. A preliminary study to find out maximum occlusal bite force in Indian individuals. Indian J Dent Res. 2014; 25(3): 325-330, doi: 10.4103/09709290.138330, indexed in Pubmed: 25098989.

9. Jiang $Y Y$, Sun $L$, Wang $H$, et al. Three-dimensional cone beam computed tomography analysis of temporomandibular joint response to the Twin-block functional appliance. Korean J Orthod. 2020; 50(2): 86-97, doi: 10.4041/ kjod.2020.50.2.86, indexed in Pubmed: 32257934.

10. Kilic N, Kiki A, Oktay H. Condylar asymmetry in unilateral posterior crossbite patients. Am J Orthod Dentofacial Orthop. 2008; 133(3): 382-387, doi: 10.1016/j.ajodo.2006.04.041, indexed in Pubmed: 18331937.

11. Lippold C, Kruse-Losler B, Danesh G, et al. Treatment of hemimandibular hyperplasia: the biological basis of condylectomy. Br J Oral Maxillofac Surg. 2007; 45(5): 353-360, doi: 10.1016/j.bjoms.2006.10.011, indexed in Pubmed: 17145124.

12. McDonnell $D$, Reza Nouri M, Todd ME. The mandibular lingual foramen: a consistent arterial foramen in the middle of the mandible. J Anat. 1994; 184 (Pt 2): 363-369, indexed in Pubmed: 8014127.

13. Meikle MC. The role of the condyle in the postnatal growth of the mandible. Am J Orthod. 1973; 64(1): 50-62, doi: 10.1016/0002-9416(73)90280-7, indexed in Pubmed: 4577167.

14. Miyazaki M, Yonemitsu I, Takei M, et al. The imbalance of masticatory muscle activity affects the asymmetric growth of condylar cartilage and subchondral bone in rats. Arch Oral Biol. 2016; 63: 22-31, doi: 10.1016/j. archoralbio.2015.11.020, indexed in Pubmed: 26669214.
15. Moss ML, Rankow RM. The role of the functional matrix in mandibular growth. Angle Orthod. 1968; 38(2): 95-103, doi: 10.1043/0003-3219(1968)038<0095:TROTFM>2.0. CO;2, indexed in Pubmed: 5239131.

16. Nakano H, Maki K, Shibasaki $Y$, et al. Three-dimensional changes in the condyle during development of an asymmetrical mandible in a rat: a microcomputed tomography study. Am J Orthod Dentofacial Orthop. 2004; 126(4): 410-420, doi: 10.1016/j.ajodo.2004.04.016, indexed in Pubmed: 15470344.

17. Neff $A$, Cornelius $C P$, Rasse $M$, et al. The Comprehensive AOCMF Classification System: Condylar Process Fractures - Level 3 Tutorial. Craniomaxillofac Trauma Reconstr. 2014; 7(Suppl 1): S044-S058, doi: 10.1055/s-00341389559, indexed in Pubmed: 25489390.

18. Rhodes G, Proffitt F, Grady J, et al. Facial symmetry and the perception of beauty. Psychon Bull Rev. 1998; 5(4): 659-669, doi: 10.3758/bf03208842.

19. Sella-Tunis T, Pokhojaev A, Sarig R, et al. Human mandibular shape is associated with masticatory muscle force. Sci Rep. 2018; 8(1): 6042, doi: 10.1038/s41598-018-24293-3, indexed in Pubmed: 29662127.

20. Taneja VK, Kumar GA, Farishta S, et al. An assessment of skeletal craniofacial asymmetry in South Indian population. J Contemp Dent Pract. 2012; 13(1): 80-84, doi: 10.5005/ jp-journals-10024-1100, indexed in Pubmed: 22430699.

21. Tecco S, Saccucci M, Nucera R, et al. Condylar volume and surface in Caucasian young adult subjects. BMC Medical Imaging. 2010; 10(1): 28, doi: 10.1186/1471-2342-10-28.

22. Tiwari S, Nambiar S, Unnikrishnan B. Chewing side preference - Impact on facial symmetry, dentition and temporomandibular joint and its correlation with handedness. J Orofacial Sci. 2017; 9(1): 22, doi: 10.4103/jofs.jofs_74_16.

23. Von den Hoff JW, Delatte M. Interplay of mechanical loading and growth factors in the mandibular condyle. Arch Oral Biol. 2008; 53(8): 709-715, doi: 10.1016/j. archoralbio.2008.03.002, indexed in Pubmed: 18395696.

24. Watanabe H, Mohammad Abdul M, Kurabayashi T, et al. Mandible size and morphology determined with CT on a premise of dental implant operation. Surg Radiol Anat. 2010; 32(4): 343-349, doi: 10.1007/s00276-009-0570-3, indexed in Pubmed: 19812884.

25. Xie Q, Yang C, He D, et al. Will unilateral temporomandibular joint anterior disc displacement in teenagers lead to asymmetry of condyle and mandible? A longitudinal study. J Craniomaxillofac Surg. 2016; 44(5): 590-596, doi: 10.1016/j.jcms.2016.01.019, indexed in Pubmed: 27021265. 